Die als Vergleich dienenden Versuche mit Zucker und anderen Nichtelektrolyten fielen aus verschiedenen Grinden und besonders aus dem Umstande, dab sie allem Anschein nach von. der Gerbsäure incht absorbiert werden, anders aus als wir erwartet hätten.

Doch beweist die zweite Reihe von Versuchen in einwandfreier Weise, dath die Kaliumchlorid-, -sulfat- und -phosphatlösungen auch unter Zugabe von erheblichen Gummi- und Dextrinmengen denselben Gefrierpunkt beibehalten; und die bei der Gerbsäure auftratenden
Unregelmäßigkeiten sind einer Brklärung zugänglich ohne Annahme irgendeines Einflusses auf die elektrolytische Dissoziation. Somit glauben wir, trotz aller möglichen Einschränkungen, schließen zu können, daß die Kolloidsubstanzen keinen Einflub auf die elektrolytische Dissoziation der Salze ausüben.

Diese Folgerung steht mit der Auffassung im Einklang, daB die Kolloide keine wahren Lösungen, sondern einfach Suspensionen bilden.

Roma. Istituto Chimico della $R$. Università.

\title{
Laterit, seine Betrachtung im Lichte der Kolloidchemie.
}

Von A. Luz (Stutigart).

(Fingerangen am 26. November 1913

Ueber wenige Stoffe im Gebiet der Mineralogie und Petrographie gingen und gehen z. T. heute noch die Ansichten der einzelnen Forscher so auseinander wie ubber Laterit, und wenige waren einem solchen Wandel in der Erklärung unterworfen, wie gerade er, der schon seit einem Jahrhundert die Gedanken der tropischen Reisenden und Geologen bewegt hat.

Wie fast nirgends in der Petrographie macht sich gerade bei Laterituntersuchungen der Mangel einer straffen; einheitlichen Definition fühlbar, die von allen Forschern in gleicher Weise angewendet wurde. Die Ursache dieses Mangels, der zum Teil heute noch weit auseinandergehenden Ansichten über den Laterit, mag nicht zum mindesten darin liegen, daB die einzelnen Forscher durch die Untersuchung der verschiedenartigsten, grobenteils gar nicht von ihnen selbst gesammelten Verwitterungsprodukte, die alle unter der Bezeichnung "Laterit " registriertwurden, notwendigerweise $\mathrm{zu}$ den verschiedensten Ansichten und Definitionen gelangen muften.

Dadurch hauptsächlich ist es gekommen, daB man in der Literatur über die Eigenschaften des Laterits sehr weit auseinandergehende Angahen findet. Denn der Name "Laterit" wurde in den Tropen allmählich auf alles angewandt, auf die verschiedensten Böden, wenn sie nur einigernaben rot oder auch nur gelb aussahen, wodurch auch dem Laterit eine viel gröbere Verbreitung zugeschrieben wird, als sie ihm wohl tatsächlich zukommt. Nach einer Schätzung von A. v. Tillo') bedeckt der Laterit in Afrika 49 Proz., in Asien 16 Proz. und in Südamerika 43 Proz. des Bodens.

1) A, v. Tilio. Die geogr. Verteilung v. Grund a. Boden, Petermann's geogr. Mitt. 39, 17 (1893).
Zwei charakteristische Beispiele nur mögen dieses. Wirnis, das in der Lateritliteratur geherrscht hat, beleuchten: So bezeichnen die einen den Laterit als gänzlich unfruchtbar, während andere ilun zu den fruchtbarsten Bodenarten stellen. Oder die einen halten eine Rotfärbung, hervorgerufen durch Eisenverbindungen, auch die Anwesentieit von Eisenoxydkonzentrationen als ein Hauptkennzeichen des Laterits, wie das $z$. B. besonders S. Passarge ${ }^{2}$ ) tut, während diese Eigenschaft den andern als zum Charakter des Laterits nebensảchlich erscheint.

Sehr interessant ist der Wechsel der Lateritanschauungen, der sich im Laufe des vorigen Jahrhunderts und namentiich um die Jahrhundertwende vollzog, der deshalb in kurzen Zijgen gezeichnet werden soll ${ }^{3}$ ).

Die erste Beschreibung finden wir bei dem indischen Geologen Buchanan, der dem Stoffe auch nach seiner dortigen Verwendung zu Ziegelsteinen $(=$ later) seinen heute noch gültigen Namen "Laterit" gab. Er und seine indischen Fachgenossen schrieben dem Laterit vulkanische Entstehung zu und stellten ihn deshalb zum "Trapp", unter welcher Bezeichnung man damals Melaphyr, Grünstein, Mandelstein, Klingstein (Phonolith) und Basalt zusammenfuBte, und den man als geologische Formation zwischen das Unter- und Oberkarbon einschob. - Andere, die scion etwas weiter blickten,

2) S. Passarge, Ueber Laterite u. Roterden in Afrika u. Indien, Bericht des internat. Geol.-Kongresses (Lonion 1895).

b) Ausfuhlicheres findet sich aus letzter Zeit besonders bei W. Meigen, Laterit, Geol. Rundschau 1911, 197 u. 198, u. dessen Schtiler R. Lenz, Chem. Untersuchung über Laterite, Inaug:- Diss. (Freiburg i. Br. 1908). 
hielten den Laterit für eine besondere Form, die den "Trapp" bedecke.

An der vulkanischen Entstehung wurde längere Zeit noch festgehalten, auch wurde er für ein Produkt von Schlammeruptionen gehaiten, und Carter hatte noch die Ansicht, daB der Eisengehalt der Laterite von eisenhaltigen Gasen, Dämpfen oder Gewässern stamme, die die Eruptivgesteine durchtränkten.

In der Mitte des yorigen Jahrhunderts nun wurde er für sedimentär gehalten, von den einen als Süfiwasser-, von anderen dagegen als Meeresablagerung. Als eine besondere Formation wurde er dann von Foote zwischen Tertiär und Quartär eingeschoben.

Erst im Jahr 1853 erkannten ihn verschiedene Forscher, wie Kelaart, Clark und Young, als Zersetzungsprodukt kristalliner Gesteine auf chemischem Wege. Doch sollte noch eine längere Zeit verstreichen bis diese richtige Anschauung allgemeine Anerkennung erlangte.

Besonders erwähnenswert ist in dieser Richtung die 1895 aufgestellte Laterittheorie von S. Pass a $\mathrm{g} \mathrm{e}^{4}$ ), det als Hauptcharakteristikum das im Laterit vorhandene Eisenoxydhydrat ansah; und zwar unterschied er zwischen einem roten Eisenoxydhydrat, das sich leicht bei Gegenwart von Salpetersäure, nicht bei Anwesenheit von Humussäuren und daher leicht in den Tropen, schwieriger bei uns, bilde, und einem gelben Eisenoxydhydrat, das für unsere Gehängelehme charakteristisch sei. Dabei könne das rote Eisenoxydhydrat durch hâufige Austrocknung und Erhitzung aus den gelben entstanden sein. Durch diese Unterscheidung suchte er eine Beschrankung des Laterits auf die Tropen zu erklären. Als Eritstehungsursachen machte er demnach Salpetersätriebidung und Abwesenheit von Humussäuren verantwortich.

Damit war jedoch das eigentliche Wesen des Laterits noch nicht voll aufgedeckt. Ein Hauptschiritt hierzu wurde von M. Bauer wenige Jahre später (1898) getan, der in Laterit die Anwesenheit von freiem Tonerdehydrat $\left(\mathrm{Al}_{2} \mathrm{O}_{3} .3 \mathrm{H}_{2} \mathrm{O}=\mathrm{Hydrargillit}\right)$ feststellte, entstanden durch eine Zersetzung tonerdehaltiger Mineralien, wie z. B. Feldspat, Augit, Homblende a. a., und zwar durch alkalische Lösungen, welche Ergebnisse durch eine Nacliprifung durch J. M. van Bem melen Bestatigung erhielien.

) S. Passazge, loc. cit. 2 .

5) M. Baner, a) Beitrăge zur Geologie der Sey. schelien (N. J. f. M U. P. 1898); b) Beitrac zur Kenntuis des Laterits, insbes. dessen von Madagaskar $(\mathrm{N} . \mathrm{J}$. $\mathrm{f}$. M. usw, 1907. Fastband).
Demgegenüber gelangt jedoch $D \mathrm{u}$ Bois ${ }^{6}$ ) zu einer vollkommen anderen Ansicht, indem er eine Zersetzung der gebirgsbildenden Gesteine durch Schwefelsäure, entstanden durch Oxydation von reichlich vorhandenem Schwefelkies, annimmt und unter Laterit alle Verwitterungsprodukte verstebt, bei denen die im Gestein enthalteneri Silikate zu sekundären Silikatverbindungen, sekundärer Kieselsäure und Tonerdehydrat, die Eisenverbindungen zu lebhaft gefärbten, meist roten Eisenoxydverbindungen umgewandelt und angehäuft werden durch chemische und mechanische Zersetzting. Gesteine ohne Schwefelkies können nach ihm demnach keinen Laterit bilden. Dieser Theorie gegenüber bemerkt W. Meigen ${ }^{7}$ ), daf auf diese Weise kein echter Laterit, sondern tonähnliche Produkte entstehen würden.

Eine andere Theorie von T.H.Holland nimmit eine Aufschliefung der Tonerdesilikate durch Salpetersäure erzeugende Baktetien an, die nur bei tropischen Temperaturen existieren könnten (die aber bis heute noch nirgends festgestellt werden kounten) und Wegtührung der freiwerdenden Kieselsäure durch gleichzeitig entstehende alkalische Lösungen, ahnlich wie $M$. Ba uer.

W. Meigen, der auch für die nächste Zeit eine neue lateritarbeit angekündigt. hat, der man wohl mit berechtigtem Interesse entgegensehen dari, kommt nach Zusanmenstellung und Betrachtung aller bekannten Tatsachen $z u$ folgender Definition: „Die Laterite sind die in den Tropen vorkammenden, infolge ihres hohen $\mathrm{Ge}$ haltes an Eisenoxyd meist tiefrot gefärbten Verwitterungsprodukte sehr verschiedenartiger Gesteine. Der Eisengehalt ist zuweilen so erheblich, da $B$ es zur Bilang zelitiger oder schlackenartiger Konzentrationen von Braun- oder Roteisenerz kommt, die manchmal eine recht beträchtliche Größe erreichen. Die Lateritisierung reicht oft bis in große Tiefen, da die zersetzten Gesteine durchlassig werden und dadurch das. immer tiefere Eindringen der atmosphärischen Sickerwässer ermöglichen. Der Laterit besteht im wesentlichen aus Tonerdehydraten, namentlich aus Hydrargillit, meist gemischt mit Eisenoxyd oder Brauneisenerz. Seine Entstehung ist darauf zurückzuführen, dap in den Tropen wegen der geringen Menge von Humusstofen im Boden die hydrolytische Wirkung des Wassers auf die Sili-

6) D n Bois, Beitrag zur Kenntnis der surinamic. Laterit- u. Schutzrindenbildung, Tschermaks min. i. petr. Mitt. 22, $1-61(1903)$.

7 W. Melgen, Laterit, Geol. Rundschau 1911 . 197 น. 198. 
kate rein zur Geltung komınt, während die Verwitterung im gemäBigten Klima in erster Linie unțer Mitwirkung von Kohlensäure erfolgt."

Ein beständiges Streitobjekt bei den Lateritforschungen der letzten Jahrzehnte bildete immer die Frage, ob neben dem freien Tonerdehydrat, dem Hydrargillit, auch gleichzeitig Tonerdesilikat (Kaolin und Ton) vorkommen. Es ist łür die späteren Ausführungen unerläBlich, dieser Frage, wenn auch so kurz als möglich, näherzutreten.

M. B auer ${ }^{8}$ ) bejaht diese Frage, wenn er sagt: „Neben den Aluminiumhydroxyden (Hydrargillit) können aber auch Aluminiumhydrosilikate (Kaolin usw.) gebildet werden", und "Die Lateritisierung führt häufig nicht zur ausschlieblichen Bildung von Aluminiumbydroxyden allein als Endpunkt des Umwandlungsprozesses, sondern daneben oder auch an ihrer Statt zur Bildung von Kaolin und anderen Aluminiumhydrosillkaten, welche die Hydroxyde dann begleiten oder ersetzen."

Zum gleichen Ergebnis kommen F.J.W a th and $H$. Warth ${ }^{9}$ ) für Indien, sowie $A$. K. Coomaraswan ${ }^{10}$ ).

Bestritten wird das Mitvorkommen von Kaolin neben Tonerdehydrat von T. H. Holl and und Rösle ril).

C. Klemen (12) erwähnt im Kongolaterit weichen plastischen Ton; ebenso bestätigt Meigen ${ }^{13}$ ) die Bildung von Kaolin neben Hydrargillit.

W. Bruhns und H. Bücking ${ }^{14}$ ) geben gleichfalls die Anwesenheit von Kaolin an.

Auch sonst wird das Mitvorkommen von Tonerdesilikaten $\left(+\mathrm{H}_{2} \mathrm{O}\right)$ im Laterit berichtet, so besonders noch von H. Ars a nda u ${ }^{15}$ ).

Seit wenigen Jahren nur hat in der Mineralogie eine Betrachtungsweise mehr und mehr Platz genommen, das ist die Erkenntnis der

g) M. Bauer, loc. cit. 4,2 b.

9) H. Warth, a) Indischer Laterit, Geoiog. Mag. 14] 10, 159 (1903); Weathered Dolerite of Rewley Regis compared with the Laterite of the Western Ghâts near Bombay, Geol. Mag. [5] 2,.21 (1905).

10) A. K. Coomaraswany, in i).

i1) T. H. Holland, On the constitution, crigin and dehydration of Laterite, Geol. Mag. [4] 10, 154 (1909), u. Chemical News 87, 256 (1903).

13) C. Klement, Laterite vom unteren Kongo, Min. u. petr. Mitt. 8, 24 (1887).

1.3) W. Meigen, EBbare Erde v. Deutsch-Nea Guinea, Ber. d. Deutsch. geol. Ges. 557 (1905).

14) W. Bruhns u. H. Bücking, Beitrag zur Kenit. nis des Laterits, Zentralbl. f. Min., Geol. u. Pal, 467 (1904).

16) H. Arsanda ax, Contributions à l'étude des formations latéritiques. Compt. rend. 149, 682 u. 1082 (1909); 149, II, $2193(1909) ; 150,1698$ (1910). großen allgemeinen Bedeutung, die den $\mathrm{Kol}$ loiden in der Mineralogie und in den mit dieser verwandten Wissenschaften zukommt.

Schon A. Breith a u t $^{16}$ ) hat sich in dieser Richtung Verdienste erworben, er ist es, der den Opal als ein Kieselsäuregel erkannte.

Nach ihm war es besonders F. Cornu, der durch seine zahlreichen epochemachenden $\mathbf{A b}$. bandlungen: "Ueberdie Verbreitung und Bedeutung von Gelen im Mineralreich* den Mineralogen und Geologen auf ganz neuartige, fast unbeschrittene, verheiBungsvolle Wege führte. Und in der Tat verspricht die Anwendung der íehren dieser ebenfalls noch ziemlich jungen.Wissenschaft, der Kolloidchernie viele komplizierte Verhältnisse und Erscheinungen nicht nur der Mineralogie, sondern auch der Geologie, Erzlagerstättenlehre und namentlich der. Bodenkunde, in ein wesentlich verständlicheres Licht zu rücken.

Besonders nachdem $E$. Dittler und $C$. Doelte $r^{17}$ ) in mehreren Abhandlungen über Bauxit (ein dem Laterit ähnliches Produkt, das im wesentlichen aus freiem Tonerdehydrat und Fisenoxydhydrat besteht) diesen als ein typisches Kolloid erkannten, lag es nahe, auch den Laterit einer kolloidchemischen Betrachtungsweise zu unterziehen.

Die Vermutung, daB man es auch bei der Lateritisierung mit der Bildung von kolloiden Lösungen und deren Ausflockung zu Gelen zu tun habe, ist an und für sich nicht so ganz neu.

In seiner Aufzählung der gelartigen und gelverdächtigen Mineralien erwähnt Cornu ${ }^{18}$ ) auch den Laterit. Nach ihm ergibt sich der -- wenigstens ursprüngliche - Gelcharakter der Laterite daraus, dabi viele Oolithstruktur aufveisen, ferner daß sich Uebergänge zum Bauxit, einem sicheren Mineralgele, einstellen.

Neben Cornu ist es besonders noch Meigen ${ }^{19}$ ), der ebenfalls eine Kolloidentstehung der Laterite annimmt. Nach ihm geht (wie schon weiter oben ausgeführt) die Bildung der l.aterite in den Tropen überall dort vonstatten, wo reich-

18) F. Cornu, A. Breithaupt, seine Bedeutung f. Kolloidchemie u. Mineralogie, Koll.-Zeitschr, 4, 300 (1909).

17) E. Dittler u. C. Doelter, a) Zur Charakteristik des Bauxits, Zeniralbl. f. Min. usw. 19 (1912); b) Bauxit ein natütliches Tonerdehydrogel, Koll.-Zeitscht. 9, 282 (1911), u. Zentralbi. f. Min. usw. 1 u. 4 (1912); c) Bauxit oder Sporogelit? Zentralbl. f. Min. usw. 7, 193 (1913).

19) F. Cornu, Die Bedeutung der Hydrogele im Mineralreich, Koll,-Zeitschr. 4, 15 (1909).

19) W. Meigen, loc. cit. 5 . 
liches Regenwasser auf nicht durch Humus bedeckte Gesteine einwirkt. Das (nicht saure) Wasser bewirkt eine hydrolytische Spaltung der primären tonerdebaltigen Silikate; so zerfällt der Feldspat in Tonerdehydrat einerseits und Alkalisilikat andererseits, das sich wiederum in Alkalibydroxyd und freie Kieselsäure zerlegt. Alle Spaltungsprodukte liegen zunächst als kolloide Lösungen vor. Während aber kolloide Kieselsäırelôsungen durch Anwesenheit von geringen Mengen von Alkalien beständig bleiben, werden die kolloiden Tonerdelösungen und Eisenlösungen durch diese ausgeflockt, wobei durch die augenblicklich erfolgende Ausscheidung die Gesteinsstruktur erhalten biiebe. Ebenso wirkt nach Meigen die Wărne, wobei diese bis zu einem gewteen Grade durch vermehrte alkalische Reaktion des Bodens ersetzt werden könne, so dat auch in Sïdeuropa, und zwar nur auf Kalkböden (z. B. Karstgebiet), eine Art Laterit entstehen kann. Zweifelhaft läBt er noch, ob die Unwandlung in den kristallinen Hydrargillit gleichzeitig oder erst nachträglich stattfindet.

Weiterbauend auf diesen neuartigen Ansichten über die Lateritentstehung, die ein Weiterstudium auf diesem inferessanten Gebiet sehr anregen, sind von mir an brasilianischen und deutsch-ostafrikanischen Lateriten zahlreiche, eingehende Untersuchungen angestellt worden. Da ich auf Grund dieser noch nicht ganz beendeten Untersuchungen zu Ansichten gelangt bin, die vielleicht manches in ein einheitlicheres Licht rücken, soll hier vorläufig siniges mitgeteilt werden.

Eine unumgängliche Voraussetzung für erfolgversprechende Untersuchungen auf dem Gebiete der Verwitterung, demnach auch beim Laterit, muB die fortschreitende Beobachtung vom absolut frischen Gestein bis zum fertigen, stabilen Verwandlungsprodukt sein, wenn man ein möglichst genaues Bild erhalten will. Gerade beim Laterit ist diese Forderung oft leicht $\mathrm{zu}$ erfüllen, finden sich doch gern Handstücke, bei denew man frisches Gestein und fertigen Laterit in unberührtem Zusammenhang beobachten kann, und es ist klar, dat solche Stücke, bei denen der Uebergang oft messerscharf ist, so dab ein gut geführter Gesteinsschliff die ganze Umwandlungszone umfaBt, besonders eine eingehende Untersuchung anregen, wobei neben chemischen Untersuchungen, namentlich fraktionierten qualitativen und quantitativen Analysen eine genaue mikroskopische Beobachtung sowohl an Dünnschliffen als auch an Pulverpräparaten unumgänglich ist.
Bei der Feldspatumwandlung läBt sich so beobachten, daf diese sich in mebreren Stufen vollzieht. Zuerst treten in den Feldspatkristallen auf Spaltrissen und anderen unregelmäBigen Rissen, die immer mehr an Zahl zunehmen, ganz leicht bräunliche, vollständig amorphe Substanzen von unregelmäBig lappig - rundlicher Form auf, die auf Grund einer Behandiung mit Salzsäure, wobei sie unlöslich blieben, sowie durch Rückschlut aus der weitergehenden Umwandlung als ein amorphes Tonerdesilikat angesehen werden müssen. Almählich greift diese Umwandlung in den Feldspatkristallen immer mehr um sich, so dab diese mehr und mehr isotrop werden, ohne daB jedoch die Feldspatform zerstört wird.

Diese Stufe hat jedoch einen verhältnismäBig kurzen Bestand, denn als weitere Stufe ist in diesen amorphen Substanzen eine gar nicht spärliche Ausscheidung von kleineren und gröBeren rundlichen bis kugeligen Gebilden zu beobachten, die mit ihrer überaus feinwabigen Struktur neben vollkommen isotropem Verhalten eine überraschende Aehnlichkeit mit den Kieselsäuregallerten zeigen, die $O$. Büt $\mathrm{ch}\left(\mathrm{i}^{20}\right.$ ) in seinen "Untersuchungen über die Mikrostruktur künstlicher und natürlicher Kieselsäuregallerten " beschreibt und abbildet. Eine Menge von bis zu 9 Proz. löslicher Kieselsäure, die $\mathbf{n}$ i ch $\mathbf{t}$ von dem vorher beschriebenen amorphen Tonerdesilikat stammen, scheinen den Schlub zu bestätigen, dak man es hier mit der Bildung eines Kiesel. säuregeis zu tun hat.

Nach der Ausscheidung des Kieselsäuregels bleibt eine amorphe, in Salzsäure jetzt lösliche Substanz ibrig, die als ein a morphes Tonerdehydrat gedeutet werden muß. Dieses ist jedoch nur sehr spärlich zu beobachten. Seine Stelle wird fast durchweg von farblosen, vielfach radialbiätterigen, schuppigen bis faserigen, öfters fast oolithartigen, schwach licht- und stark doppelbrechenden Aggregaten von kleinen Blăttchen eingenommen, die unzweifelhaft dem $\mathrm{Hy}$ drargillit angehören. Die Analysen ergaben eine mit Temperatur, Einwirkungsdauer und Konzentration der Säure steigende Löslichkeit in Salzsäure; wie dies auch von J. M. van Bemmele $n^{21}$ ) festgestellt wurde.

20) O. Butschli, Verhandlungen des naturh.-med. Vereins zu Heidelberg, N. F. 6, 287-348.

21) J. M. van Bem me len, Beitrăge zur Kenntnis der Verwitterungsprodukte der Silikate in Ton-, vulkanischen u. Lateritboden, Zeitschr. f. anal. Chem. 42, $265 \mathrm{ff}$. (1904). 
Ganz entsprechende Umwandlungserscheinungen lassen sich bei der Umwandlung von Chlorit, Augit und Glimmer beobachten, nur tritt besonders beim Augit noch eine reichliche Ausscheidung von Eisenhydroxyd hinzu, das zumeist ebenfails amorphen Charakter aufweist.

Nicht alle untersuchten Umwandlungsprodukte sind bis zum Stadium des kristallinen Hydrargillits vorgeschritten. Viele befinden sich noch auf der Stufe des amorphen Tonerdesilikats, dessen genauere Zusammensetzung nicht zu ermitteln war, das aber nirgends in eine kristalline Modifikation umgewandelt war. Die Eisenhydroxydausscheidung war dabei schon beendigt.

Magnesium, Kalzium, Kalium und Natrium konnten in von unzersetztem Material freien Proben nicht mehr festgestellt werden: ein Beweis dafür, daß sie weggeführt worden sind.

Zur Untersuchung auf den Gelcharakter der amorphen Umwandlungsprodukte wurden besonders zwei Methoden angewendet: Färbemethoden und Bestimmung des Wassergehaltes.

Nach Vorgang von J. M. van Bemmelen ${ }^{22}$ ) wurde unterschieden zwischen "scbwächer ${ }^{*}$ und "stärker" gebundenem Wasser, wonach das "schwächer "gebundene, das über konzentrierter Schwefelsäure und bei Tenıeraturen bis zu $110^{\circ}$ entzogen wird, Absorptionswasser von Kolloiden darstellt, wăhrend das von Kristallen gebundene ( "stärkere ") erst bei höheren Temperaturen, beim Hydrargillit z. B. erst bei über $180^{\circ}$ entweicht. Auf diese Weise wurde "schwächer" gebundenes Wasser bis zu 4 Proz. festgestellt, wobei noch bedacht werden muB, daB das Wasser in kolloiden Substanzen um so stärker gebunden ist, je weniger es absorbiert ist, womit man bei den tropischen Lateritproben der dütren, ausgetrockneten Erdoberfläche wohl zu rechnen hat.

Die Färbemethoden, beruhend auf der hauptsächlich den Gelen zukommende Fähigkeit, basische und saure Farbstoffe unter Anfärbung aufzunehmen, sind in der letzten Zeit vielfach in der Gelforschung benutzt worden, wobei sich ergeben hat, dab sie mit Vorsicht zu gebrauchen sind, da sich auch kristalline Stoffe, wie kristallines Kaolin, auf diese Weise anfärben lassen.

Bei der Anwendung der Färbemethoden in der Bauxitforschung erzielten $F$. Hundeshagen ${ }^{23}$ ) und E. Dittler,. C. Doelter ${ }^{24}$ ) günstige Ergebnisse, indern sie konzentrierte

2) J. M. van Bemmelen, loc. cit.

20) F. Hundeshagen, Ueber die Anw endung org. Farbst. zur dlagnost. Färbung mineral. Substrate, N. J. f. M. usw., Beil.-Bd. 28, 335 (1909), u. Zeitschr. f. ang.
Methylenblau-Säurefuchsinlösungen wenige Minuten auf Dünnschliffpräparate einwirken ließen. F. Hundeshagen fand, daß im allgemeinen kristalline Substanzen, selbst in feinster, mechanischer Verteilung, nicht färbbar sind, dagegen verhalten sich die natürlichen oder künstlichen amorphen und kryptokristallinen Silikate stark chromatophil, vorwiegend basophil. E. Dittler und C. Doelter erbielten folgende Ergebnisse:

1. Hydrargillit und Diaspor sind nicht färbbar, höchstens, wenn sie zersetzt sind.

2. Ton und Kaolin reagieren stark basophil.

3. Kieselsäure ist als Quarzsand unfärbbar.

4. Brauneisenerz reagiert sauer und färbt sich basophil. Die Anfärbung ist geringer als beim Tonerdehydroxyd.

Man sieht also, daB die Färbemethoden schon imstande sind, einige Aufklärung zu verschaffen. Durch mehrere Versuchsreihen an Pulverpräparaten und Dünnschliffen durch Behandlung mit Methylenblaulösung konnte einmal festgestellt werden, dab das Lateritpulver bei Ueborschichtung mit Farbstofflösung diesen ziemlich reichlich unter Entfärbung der überstehenden Flüssigkeit aufzunehmen imstande ist. Bei mikroskopischer Untersuchung des gefärbten Pulvers und Vergleichung mit angefärbten Dünnschliffen, sowie mit kalter und heifer, verdünnter und konzentrierter Salzsäure behandeltem uìd dann ebenfalls mit Methylenblau angefärbtem Pulver ergab sich:

1. Das in Salzsäure unlösliche amorphe Tonerdesilikat, sowie das spärliche amorphe Tonerdehydrat werden stark gefärbt, wogegen der kristalline Hydrargillit ungefärbt bleibt.

2. Das Kieselsăuregel färbt sich ebenfalls.

3. Das amorphe Eisenhydrozyd färbt sich ebenfalls, wenn auch schwach, bei dem kristallisierten Brauneisenerz ist jedoch eine Anfärbung nicht zu beobachien.

Diese kurz angeführten Ergebnisse, die später noch weiter ausgeführt und denen dann noch einige weitere hinzugefïgt werden sollen, lassen doch schon zur Genüge ersehen, daß man es hier mit echten Gelen zu tun hat: Tonerdesilikatgel, Tonerdehydrogel, Kieselsäuregel und Eisenhydroxydgel. Aus dem Tonerdehydratgel tind dem Eisenhydroxydgel mu man sich dann die

Chem. u. Zentralbl. f. techn. Chems. 21, 2405 1. 2454 (1908).

24) E. Dittler u. C, Doelter, Bauxit ein naturl. Tonerdehydrogel, Koll.-Zeitschr. 9, 282 (1911) u. Zentralbl. 1. Min. usw. 1 u. 4 (1912) u. Zur Clanakteristik des Batuxits, Zentralbl. f. Min. usw. 19 (1912). 
kristallisierten Substanzen Hydrargillit und Limonit hervorgegangen denken, was weiter unten näher begründet werden soll.

Das Ganze noch einmal übersichtlich zusammengestellt, ergibt folgendes Umwandlungsschema:

Ursprüngliches Mg-, Fe-, Ca-, K- und Na-haltiges krist. Tonerdesilikat

I.Stufe: kolloides Tonerdesilikat
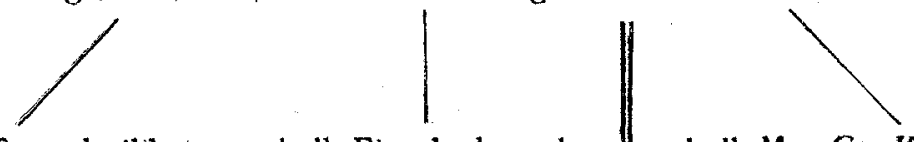

II.Stufe: koll. Tonerdehydrat koll. $\mathrm{SiO}_{2}$

koll. Eisenhydroxyd

koll. $\mathrm{Mg}-\mathrm{Ca}^{-}, \mathrm{K}-, \mathrm{Na}-\mathrm{Silikat}$ (meist gendscht mit $\mathrm{SiO}_{2^{-}}$Lós.)].

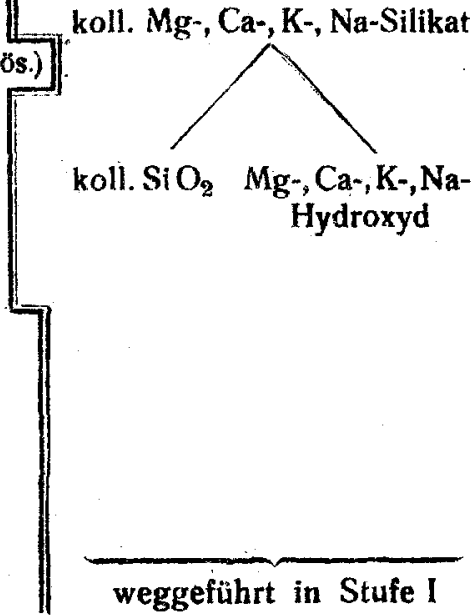

koll. $\mathrm{SiO}_{2} \quad \mathrm{Mg}-\mathrm{Ca}-, \mathrm{K}-, \mathrm{Na}-$ Hydroxyd

III. Stufe: krist. Hydrargillit

krist. Limonit

Diese Ansicht ist der von W. Meigen in vielen Zügen nicht unähnlich, nur muß ich auf Grund mannigfacher Beobachtungen eine fortlaufende Reihe vom kolloiden Tonerdesilikat uber das kolloide Tonerdehydrat zum kristallinen Hydrargillit (ev. Diaspor) annehmen, wobei die weiter auftretenden Uebergangsprodukte sich nur durch einen von den äuberen Bedingungen

- hauptsächlich Temperatur - abhängenden Wassergehalt unterscheiden können. Dazu kommt noch die bei der Spaltung des Tonerdesilikats sich ergebende kolloide Kieselsäure. Magnesium, Kalzium, Kalium und Natrium muB man sich gleich in Form von Silikatlösungen weggeführt denken, eine Spaltung in Hydroxyd und Kieselsäure findet dann erst an sekundärem Orte statt, dort oft grötere Knollen von amorpher Kieselsäure bildend. Infolge dieser Substanzwegführung muB eine Volumverminderung stattfinden, die auch immer festgestellt werden kann. Als spaltende Kraft nehme ich, ebenfalls wie Meigen, in erster Linie die hydrolytische Wirkung des humussäurefreien Wassers an, welcher Kraft überhaupt in der Natur wohl eine weit größere Bedeutung zugerechnet werden $\mathbf{m u B}$, als dies seither geschehen ist.

Dak auch die Tonerde mehr oder weniger öters der Wegführung unterliegt, läßt sich in der Lateritliteratur aus manchen Analysen aus

dem niederen Tonerdegehalt der Umwandlungsprodukte gegenüber dem des ursprünglichen Gesteins ersehen, was auch aus den von mir ausgeführten Analysen mehreremals unzweifelhaft hervorgeht.

Zu dem aus dem Umwandlungsschema Ersichtlichen muß noch ein Punkt erwähnt werden. Aus mancherlei Anzeichen, besonders im mikroskopischen Bild, mull man die Annahme einer Mischung von kolloider Kieselsäure mit Eisenhydroxyd, und zwar in wechselndem Verhältnis der beiden Komponenten machen. In mehreren Lateriten wurden nun oft viele kleine, vereinzelt beinahe Millimeter große Klümpchen von kleinen, gelbgriin durchsichtigen, anscheinend rhombischen, stark doppelbrechenden Blättchenaggregaten beobachtet. Stellenweise zeigen sie alle Uebergänge in der Farbe vom Gelbgrün bis zur schwarzen Undurchsichtigkeit. Die Kleinheit dieser vereinzelt zerstreuten Aggregate erlaubt leider keine chemische Analyse. Untersuchungen ain Dünnschliff ergaben ihre indifferente Stellung gegenüber Farbstoffen, ferner ihre Unlöslichkeit gegenüber Salzsäure. Merkwürdigerweise trat jedoch ein Gelatinieren mit der Säure ein unter Aufquellen, bei längerer Einwirkung, und die Substanz wurde jetzt der Farbstoffeinwirkung zugänglich. Das ganze Verhalten dieser Substanz, ihre Auftretungsweise, 
die offenbar erfolgende Weiteruxydation an der Luft zwingen zu der Annahme, dab die Substanz ein aus den oben erwähnten amorphen Mischungen von Kieselsäure und Eisenoxyd hervorgegangenes kristallisiertes Eisensilik at vorstellt, welcher Annahme die fraktionierten chemischen Analysen des Gesteins nicht widersprechen.

Die Annahme dieses stufenweise fortschrej. tenden. Umwandlungsprozesses bei der Lateritisierung läbst vieles in einem einheitlicheren Lichte erscheinen. Sie berechtigt eine Stellungnahme zu der früher erwähnten Streitfrage, ob bei der Lateritisierung neben Tonerdehydraten auch Tonerdesilikate entstehen. Nach obigem müssen die Lateritisierungspunkte in minde. stens drei Hauptstadien auftreten können, die hauptsächlich von Alter. Temperatur und Feuchtigkeit abhängig sein müssen. Daher kann es dann kommen, dab bei einem in der Umwandlung wenig fortgeschrittenen Laterit mehr die dem Stadium I entsprechenden Produkte, wie besonders amorphes Tonerdesilikat, gefunden werden können, was oft auch bei den hier untersuchten Proben der Fall ist. In mittleren Stadien (II) werden hauptsächlich amorphes Tonerdehydrat und im weiter fortgeschrittenen Zustand (III) wird dam hauptsăchlich kristallisierter Hydrargillit und vielleicht noch kristallisierter Diaspor entdeckt werden können.

Von Bedeutung ist dies alles auch für das Verbältnis vom Laterit zum Bauxit (beide als Gesteinsbegriffe verstanden). Schon $\mathrm{H}$. Warth ${ }^{25}$ ) sagt: "Laterite is bauxite in various degrees of purity, from the richest, wocheinite down to such specimens, in which the free alumina has entirely disappeared." Dieser Definition schlob sich dann auch $M$. B a u e $r^{26}$ ), an, ${ }_{n}$ unter der Voraussetzung, daf unter Bauxit die Aluminiumhydroxyde, vor allem Hydrargillit, verstanden wìrden".

Nach den Feststellungen von E. Dittler und $\mathrm{C}$. Doelte $\mathrm{r}^{27}$ ) besteht der Bauxit neben Eisenhydroxyden im wesentlichen aus kaolinartigen, amorphen Substanzen, kolloidem Aluminiumhydroxyd, Hydrargillit und Diaspor. Und zwar bestehen viele Bauxite hauptsichlich aus kolloidem Tonerdehydrat neben verhältnismäßig spärlichem, manchmal beinahe gänzlich fehlendem kristallisierten Tonerdehydrat, bei andern

25) H. Warth, [ndischer Laterii, Geol Mag. [4] 10, 159 (1903).

20) M. Ba uer, loc cit. $4 b$.

27) E. Dittler u. C. Doelter, Zur Charakteristik des Bauxits, Zentralbl. f. Min. usw. 19 (1912). ist es wiederum umgekehrt. Das Verhältnis von amorphem, kolloiden Tonerdehydrat zu kristallinem mag auch hier hauptsächlich vom Alter der Kolloide und der herrschenden Temperatur abhängig sein.

Versteht man unter den Bezeichnungen "Enterit" und "Bauxit" Gesteinsbegriffe, wie dies wohl nach der ganzen Natur beider anzeigt erscheint, so kann man wohl ohne Bedenken beide für identisch erklären, zumal auch jeder einzelne keine konstante Zusammensetzung aufweisen kann.

Nach Dittler und Doelter, welche die tonerdehydratreichen Massen "Alumolithe" benennen und dann uniterscheiden zwischen "KolloidAlumolithen" und "Kristalloid-Alumolithen", haben die letzteren ein hoheres Alter, indem letztere aus den ersteren hervorgehen. Dieselbe Unterscheldung mit demselben genetischen $\mathrm{Zu-}$ sammenhang läbt sich genau so beim laterit durchführen. Diese beiden Autoren haben auch durch Laboratoriumsversuche an künstlichen Tonerdehydrogelen (ebenso auch bei Eisenoxydhydratgel) gefunden, daß sich diese bei Anwendung von etwas erhöhter Temperatur nach jahrelangem Liegen der Formel $\mathrm{Al}_{2} \mathrm{O}_{3} \cdot \mathrm{H}_{2} \mathrm{O}$ (= Hydrargillit) nähern, d. h. sie werden $\mathrm{zu}$ Hydrargillit und schlieBlich durch noch mehr Wasserverlust zu Diaspor $=\mathrm{Al}_{2} \mathrm{O}_{3} \cdot \mathrm{H}_{2} \mathrm{O}$. Dic Unwandlungsgeschwindigkeit der kolloiden Tonerde in die kristalline werde je nach der Konzentration der Ausgangslösungen, der Lagerungszeit und der Erwärmung eine verschiedene sein.

An dieser Stelle mul die Frage der Umwandlungsfáhigkeit amorpher Stoffe in kristallinische kurz erörtert werden.

Neben inanchen anderen sucht P. P. von Weimarn ${ }^{28}$ ) zu beweisen, daB allein der kristalline Zustand der Materie zukommt und dab eigent. liche amorphe Körper nicht existieren. Diese sind nach ihm nichts anderes als feinste Kristall. aggregate, die mit unseren heutigen Instrumenten nur noch nicht alle als solche erkannt werden können. Doch wird gerade aus dem letzteren Grunde wenigsteris der Begriff „amorph “ vorerst noch nicht aufgegeben werdeñ können.

C. Doelte ${ }^{29}$ ) bemerkt $2 u$ dieser Frage, das die chemischen Reaktionen zur direkten krisiallinen Abscheidung nur in selteneren Fällen, nämlich bei Schmelzen, fïhre, obgleich atuch da der Fall der glasigen Abscheidung eintreten

23) P. P. v. Weimarn, Koll. Zeitschr. 3, 4 u. 5 instes. 4,36 (1909).

27) C. Doelter, Ueber die Umwand ang amorpher Korper in kristallinische, Koll.-Zeitschr. 4, 295 ff. (1909). 
kann. Bei der Mineralbildung durch Abschejdung aus wässerigen Lösungen erhaite man zumieist kolloide Stoffe, und diese mükten durch einen besonderen Prozeb in kristalline Modifikationea umgewandelt werden. Darin stimmt er mit von Weimarn überein, daB in der anorganischen Natur der amorphe Zustand der labile und und der kristalline der stabile sei, und dak der labile Zustand die Tendenz habe, sich in den stabilen umzuwandeln.

Auch Barlow ${ }^{30}$ ), im Gegensatz zu Backhuis-Roozeboom ${ }^{31}$ ), hat auf Analogien 2 wischen Kolloiden und Kristallen aufmerksam gemacht, und auch er glaubt, dab ein Zwischenzustand zwischen Flüssigkeiten und kristallinen Stoffen existiere, nämlich Flüssigkeiten mitregelmäBigersymmetrischerAnordnung der Moleküle. Dieselbe Ansicht entwickelt auch F. Cornu ${ }^{\mathbf{3 2}}$ ).

Nach C. Doelter ${ }^{33}$ ) ist für die Umwandlung amorpher Stoffe in kristallinische eine Reihe äuBerer Einflüsse nötig, wie z. B. Stoß, Pressung, Belichtung, Bestrahlung mit Radiumstrahlen, Schütteln, Temperaturerhöhung u. a., sowie die Kristallisationsgeschwindigkeit der betreffenden Substanz.

Bezüglich des Aluminiumbydroxyds meint von Weimarn, dab es ziemlich leicht kristallisiere, und dab die Tatsache, daß es gewöhnlich gallertartig erhalten wird, nur davon abhănge, daB die Löslichkeit des Aluminiumhydroxyds in reinem Wasser nur gering ist. Er hält dann auch alle Niederschiage von $\mathrm{Al}_{2} \mathrm{O}_{3}, 3 \mathrm{H}_{2} \mathrm{O}$ für kristallin.

C. Doelte ${ }^{34}$ ) hat in dieser Richtung verschiedene Laboratoriumsversuche angestellt, die für die oben entwickelte Ansicht über die Lateritisierung von grundlegender Bedeutung sind, weshalb sie kurz angeführt werden sollen. Ein gelatinoser Niederschlag von Eisenoxydhydrat mit allen Merkmalen des kolloiden Stoffes, zeigt sehr grobe Tendenz zur Kristallisation.' Man braucht nämlich nur auf dem Wasserbad einzudampfen und einige Tage zu erwärmen, so hat man schwarze Kristalle von Göthit $\left(\mathrm{Fe}_{2} \mathrm{O}_{2} \cdot \mathrm{H}_{2} \mathrm{O}\right)$, bei noch längerem $\mathrm{Er}$ hitzen kann sich sogar Hämatit $\left(\mathrm{Fe}_{2} \mathrm{O}_{3}\right)$ unter Wasserentziehung bilden.

30) Barlow, Analogien zwischen Kristallen u. Kolloiden, Zeitschr. I. Krist. 29, 566 (1898).

31) Bakhuis-Roozeboom, Heterogene Gleichgewichte 1, 153 (1901).

83) F. Cornu, Zur Theorie der Kolloide, Koll.Zeitschr. 4, 305 (1909).

3) c. Doclter, loc. cit.

34) C. Doeiter, loc. cit.
Eisen hydroxydgel, hergestellt aus Ferrosulfat und Kalilauge, wird nach 88 Tagen in der Schüttelmaschine ganz kristallin, ebenso Alu m iniu m hydroxyd.

Beim Aluminiumhydroxyd bildete sich bei den einen Versuchen $\mathrm{Al}_{2} \mathrm{O}_{3} \cdot 1 \mathrm{H}_{2} \mathrm{O}$, bei den anderen $\mathrm{Al}_{2} \mathrm{O}_{3} \cdot 3 \mathrm{H}_{2} \mathrm{O}$. Der letztere Fall war der häufigere. Doelt e r beobachtete dabei lebhaft glänzende Schüppchen, etwas bläulich im durchfallenden Licht, mit dem Brechungskoeffizient 1,544. Ferner zeigten sie lebhafte Polarisationsfarben, waren in Salzsäure schwer löslich, unschmelzbar, verloren erst beim Glähen ihr Wasser. Das sind lauter Eigenschaften, die für den Hydrargillit charakteristisch sind. Man hat es demnach in dem einen Falle mit der Bildung von Diaspor, in dem anderen, hatufigeren mit der Bildung von Hydrargillit zu tun.

Die kolloide Tonerde wandelt sich sehr gern in die kristalline Modifikation um, schon beim Stehenlassen bei der verhältnismäßig niederen Temperatur von $60^{\circ}$.

Wendet man die Ergebnisse dieser Laboratoriumsversuche an auf die natürlichen Verhältnisse, so findet dadurch die oben getroffene Annahme der Entstehung des Hydrargillits aus einem Tonerdehydrogel ihre Bestätigung.

Damit gewinnt auch wieder die frühere Vermutung Bauer's ${ }^{35}$ ) von Diaspor im fertigen Laterit an Wahrscheinlichkeit, wogegen $J$. M. va n B emmele $n^{36}$ ) neben anderen Gründen hauptsåchlich den anführte, daf für die Bildung von Diaspor neben Hydrargillit kein . Beweis vorhanden sei, der aber durch die Untersuchungen von Doelter offenbar erbracht ist. $J$. $M$. van Bemmelen nimmt dafür amorphe Verbindungen von Alaunerde mit unbestimmten Mengen von Kieselsăure und Wasser an, welche Annahme ja auch in dem oben entwickelten Umwandlungsschema nicht ausgeschlossen ist.

Weiter unten gibt er dann selbst, dem der Hydrargillit ebenfalls eine letzte Umbildung einer amorphen Substanz in eine kristalline zu sein scheint, wobei $3 \mathrm{Mol}$. Wasser chemisch gebunden werden, die Möglichkeit von Umständen $z u$, bei denen der kristalline Hydrargillit wieder Hydratwasser verliert und in kristallinen Diaspor übergeht.

Mit Sicherheit ist der Diaspor bis jetzt noch in keinem Laterit festgestellt worden, aber die Möglichkeit seiner Bildung kann nach obigem nicht völlig geleugnet werden.

5) M. Bauer, loc. cit. 4.

66) J. M. van Bemmelén, loc. cit. 13. 
Bei dieser Frage der Umwandlung von kolloidem Tonerdehydrat in kristalinen Hydrargillit, ev. auch in Diaspor, ist bezüglich des Temperaturfaktors wohl ein bis jetzt noch kaum bedachter Umstand zu erwägen. Die meisten Laterituntersuchungen haben sich mit an losen Gesteinsblöcken und Felswänden gebildeten Lateritrinden oder doch mit direkt der unbedeckten Erdoberiläche entnommenen Proben beschäftigt, und gerade diese Untersuchungen stellten eine besonders relchliche Anwesenheit des Hydrargillits fest ${ }^{37}$ ). Man darf nun zweifellos annehmen, daB gerade an solchen Stellen, die bei direkter Bestrahlung durch die Tropensonne zeitweilig Temperaturen annehmen müssen, welche die bei den Laboratoriumsversuchen angewandten Temperaturen von $60^{\circ}$ mindestens erreichen, diese im Verein mit dem Zeitfaktor den labilen amorphen Zustand des Tonerdehydratgels unzweifelhaft zugunsten des stabilen kristallinen ändern müssen.

Der Unterschied der Temperatur wird woh! auch ein wesentlicher Faktor dafür sein, daß man in unseren Klimaten in dem Bauxit mehr ein amorphes Tonerdehydrat, in den Tropen im Laterit mehr den kristallinen Hydrargillit, besonders an Oberflächenstellen, antrifft.

Interessant in diesem Zusammenhang ist auch die Beschreibung eines Basaltlaterites von Java, der nach E. C. J. Mo h r ${ }^{38}$ ) zur Halfte aus Hydrargillit, zur anderen Hälfte aus einer amorphen, kolloiden Masse besteht, in der die Kieselsäure großenteils an Eisenhydroxyd (vgl. dies mit den oben angeführten Mischungen von Kieselsäure und Eisenhydroxyd) und vielleicht an etwas Aluminiumoxyd gebunden ist.

Solche Beispiele des Zusammenvorkommens von kolloidem und kristallinem Tonerdehydrat, wobei letzteres aus ersterem hervorgegangen sein mut, lassen sich aus der Laterit- und Bauxitliteratur noch reichlich vermebren.

Als eine weitere Illustration des oben Ausgeführten mag noch einiges aus der Biographie des Hydrargillits dienen, wie diese besonders in dem. "Handbuch der Mineralogie" von C. Hintze und in "Lacroix, Min. de France", zusammengestellt ist. Die dort aufgeführten Beschreibungen seiner Vorkommnisse lassen drei verschiedene Hauptausbildungsweisen erkennen:

87) Vgl. z. B. die Untersuchungen von $M$. B a uer, loc. cit. 4 .

89) E. C. J. M o hr, Ueber die Zusammensetzung v. gelbem, aus Basalt entstanderiem Laterit, Chem. Centr. 1. 1007 (1911).
Ia. Selbständige Kristalle;

Ib. Aggregate von kristallinem Charakter;

Il. Amorpher Zustand von kolloidem Charakter.

la. Selbständige Hydrargillitkriställchen wurden verschiedentlich aufgefunden, z. B.:

1. im Vagelsberg in Grobhessen im umgewandelten Plagioklasbasalt, als bis $0,1 \mathrm{~mm}$ große Kriställchen;

2. als Seltenheit $1-2 \mathrm{~mm}$ grofe durchscheinende, grïne bis blaugtüne Kristalle in Drusen eines Granatfelses bei Gleinitz-Jordansmijhl in Schlesien;

3. als meist unregelmäbige, doch auch mebbar sechsseitige Tateln von $2-3 \mathrm{~mm}$ Größe bei $0,5-0,7 \mathrm{~mm}$ Dicke, meist verzwillingt, vom Langesundfjord in Norwegen;

4. als verzwillingte Blättchen im Laterit vom Mont Kakonlima in Guinea.

Ib. Häufiger scheint der Hydrargillit in rundlichen, nierenförmigen bis kugeligen, faserigen Aggregaten aufzutreten, z. B.:

1. als grauweifle kugelige, immer radialblätterige Aggregate bei Klein-Tresny in Böhmen;

2. als radialfaserige, seiden- bis perlmutterglänzende Aggregate aư Kluftflächen von Quarz im Glimmerschiefer im Gmundener Graben bei Lieseregg in Kärnten:

3. als warzige, laserigblätterige Aggregate aus Neu-Kaledonien;

4. als traubige oder nierige, auch zapfenförmige oder plattige, knollige Bildungen, zuweilen versteckt faserig, von der Insel St. Marie bei Madagaskar ${ }^{39}$ ).

Aehnliche faserige, strahlige, warzige, nierige, kugelige Aggregate werden noch öfters angegeben, doch mögen die angeführten Beispieie hierfür genügen.

II. Auch in amorphem Zustand wird der Hydrargillit beschrieben, z. B.:

1. amorph, halloysitähnlich in einem Laterit von Bhekowli, Ostindien;

2. vollkommen amorph, leicht an der Zunge haftend, durch Reiben mit dem Finger Glanz annehmend, weiblich wie Rahm, mit einem Stich ins Rote, van den Paini Hills bei Madras, Ostindien.

Endlich ließe sich noch eine vierte Gruppe von Vorkommnissen zusammenstellen, nämlich solche, wo sich die drei aufgeführten verschiedenen Ausbildungsweisen zugleich vorfinden.

89) M. Bauer, loc. cit. 4. 
Diese Zusammenstellung einiger typischer Hydrargillitvorkommnisse nach obigen Gesichtspunkten redet eine lebendige Sprache für die kolloide Entstehung des Hydrargillits, wenn man sich auf die Seite der Ansicht von F. Corn a ${ }^{40}$ ) stellt. nach der für kristalline Mineralien, die sich aus Gelen gebildet haben, häufig matter Glanz, radialfaserig-schalige oder oolithische Struktur, manchmal seifenartige oder erdige Beschaffenheit und, wenn es sich um freie Formen handelt, traubige, kugelige oder nierenförnige Oberfläche charakteristisch ist.

Wichtig und für die Erklärung gewisser Erscheinungen in den Lateriten und Bauxiten verwendbar sind die neueren Untersuchungen von R. E. Lieseg $\mathrm{ng}^{41}$ ) über wachsende Kieselsäuregele, wonach Gele von einer bestimmten Stelle aus "weiterwachsen" können. In Lateriten und Bauxiten werden offters kleinere und gröBere Knollen von amorpher bzw. mikrokristalliner Kieselsäure, eine Art von Hornstein-, Opal.- und Achatknollen, erwăhnt, deren Bildung sich demnach, wie schon weiter oben angedeutet, durch "Weiterwachsen" des bei dem UmwandlungsprozeB freiwerdenden Kieselsh̆uregels zwanglos erklären läßt. Eine eventuelle Bänderung dieser Knollen läßt sich analog den Li e segang'schen Schichtungen in Gallerten verstandlich machen.

Auf ähnliche Weise mögen dann auch die in den Lateriten erwähnten gröteren Eisen erzkonzentrationen, sowie die freien, knolligen Formen des amorphon, spüter faserigkristallinen To n crde hydrat s entstanden sein.

Zum Schlusse noch einige Worte über den Laterit in seiner bodenkundlichen Bedeutung. Dies ist namentlich für uns Deutsche insofern von Bedeutung, als unsere ostafrikanische Kolonie anscheinend auf weiten Flächen, nantentlich den Hochflächen aus alten kristallinen Schiefern (Gneis, Phyllit und Glimmerschiefer), von diesem Umwandiungsprodukt bedeckt ist.

Es wurden von mir mehrere Lateritbodenproben, einer Hochfläche vom Norden der Kolonie

40) F. Cornu, Die Bedeatung der Hydrogele in Mineralreiche, Köl.-Zeitschr. 4, 15 (1919).

4) R. E. Liesegang, Wachsende Kieselsaregele, Koll. Zeitschr. 10, 273 (1912). entstammend, auf der Plantagenwirtschaft betrieben werden sollte, untersucht, wobei sich herausstellte, daß der Boden hauptsächlich aus $\mathrm{SiO}_{2}, \mathrm{~F}_{4} \mathrm{O}_{3}, \mathrm{Al}_{2} \mathrm{O}_{3}$ und ca. 13 Proz. $\mathrm{H}_{2} \mathrm{O}$ bestand, welche sich auf die Mineralien Quarz. (krist.), Hydrargillit, spärliches amorphes Tonerdesilikat und kristallines Brauneisenerz verteilen. Magnesia, Kalk und die Alkalien fehlen vollständig.

Schon diese kurze Skizze genügt, um zu zeigen, da $\dot{B}$ der Boden keine ungünstigeren Eigonschaften mehr besitzen könnte: Die für die Vegetation unentbehrlichen Nährstoffe werden in die Tiefe weggeführt, die unbrauchbaren, unlöslichen bleiben zurück und keine von unten nach oben sich vollziehende Wasserbewegung bringt die Nährsalze in den ausgetrockneten Hochflächen wieder an die Oberfläche. Vor allem fehlt es, entsprechend der ganzen oben entwickelten Lateritisierung, den Lateritböden anscheinend an jenen gelartigen Stoffen, die in den feuchteren, humusbedeckten Böden imstande sind, durch ihren besonderen Bau die Nährsalzlösungen adsorptiv an der Bodenoberfläche festzuhalten.

Wenn man also mit Recht die typischen Lateritböden in bodenkundlicher Hinsicht zu den denkbar ungünstigsten zählen muB, so darf doch die schon in der Einleitung erwähnte Behauptung einiger Forscher, daß der Laterit auch sehr fruchtbar sei, vielleicht nicht blok aus einer absoluten Verwechslung entstanden gedacht. werden, wennschon letztere Annahme grohe Wahrscheinlichkeit besitzt. Es wäre theoretisch denkbar, daB ein noch nicht bis zum letzten Ende fortgeschrittener, sondern hauptsächlich aus amorphen Produkten noch bestehender Lateritboden imstande ist, die ihm natürlich oder künstlich gebotenen Nährsalze festzuhalten, welche Făhigkeit einem hauptsăchlich aus kristallinen Substanzen bestehenden Lateritboden abgehen muß. Demnach wäre also dle Tendenz der Lateritböden, in mehr und mehr kristaliinen $\mathrm{Zu}$ stand überzugehen, ais der Hauptfaktor anzu. sehen, der sie leider zu den Stiefkindern der Verwitterung stellt.

Stuttgart, November 1913.

Technische Hochschule. 